\title{
Pure general gauge mediation for early LHC searches
}

\author{
Steven Abel, ${ }^{a, b}$ Matthew J. Dolan, ${ }^{c}$ Joerg Jaeckel ${ }^{a}$ and Valentin V. Khoze ${ }^{a}$ \\ ${ }^{a}$ Institute for Particle Physics Phenomenology, Durham University, \\ South Road, Durham DH1 3LE, United Kingdom \\ ${ }^{b}$ TH Division, Physics Department, CERN, \\ CH-1211 Geneva 23, Switzerland \\ ${ }^{c}$ DAMTP, CMS, University of Cambridge, \\ Wilberforce Road, Cambridge, CB3 OWA, United Kingdom \\ E-mail: s.a.abel@durham.ac.uk, m.j.dolan@durham.ac.uk, \\ joerg.jaeckel@durham.ac.uk, valya.khoze@durham.ac.uk
}

ABStRACT: We present benchmark points for Pure General Gauge Mediation (GGM) models specifically optimised for early LHC searches. The pure GGM set-up is as defined in our previous paper arXiv:0910.2674: namely we adopt the minimal set-up in which $B_{\mu}$ is generated only through gauge interactions, and as a result $\tan \beta$ is a prediction rather than an input. The only input parameters are messenger masses together with two independent scales which generate gaugino and scalar masses. The parameter space favoured by current experimental data includes an interesting region with light gluinos $\left(m_{\tilde{g}} \lesssim 500 \mathrm{GeV}\right.$ and relatively heavy squarks) and a bino-like NLSP where early discovery is likely. We also find interesting regions of parameter space where the NLSP is a stau or stau/neutralino co-NLSP and the squark masses are relatively low. We present benchmark points which are typical for each of these three regions, and give the spectrum, branching ratios, and also the overall $2 \rightarrow 2$ cross-sections. This data in SLHA format can be found at http://www.ippp.dur.ac.uk/ SUSY.

Keywords: Supersymmetry Breaking, Beyond Standard Model, Supersymmetric Standard Model

ARXIV EPRINT: 1009.1164 


\section{Contents}

1 Introduction $\quad 1$

2 Benchmark points with light gluinos for early LHC discovery 4

3 Survey of NLSP phenomenology 10

$\begin{array}{ll}3.1 \text { Stau and co-NLSP Benchmark points } & 14\end{array}$

$\begin{array}{lll}4 & \text { Conclusions } & 17\end{array}$

\section{Introduction}

Ref. [1] introduced a novel framework suitable for discussing and analysing general models of gauge mediation in a model-independent way. The so-called General Gauge Mediation (GGM) paradigm [1] is defined by the requirement that the Minimal Supersymmetric Standard Model (MSSM) becomes decoupled from the hidden SUSY-breaking sector in the limit where the three MSSM gauge couplings $\alpha_{i=1,2,3}$ are set to zero. Since no other parameters participate in the coupling of the two sectors, we call this strict interpretation of gauge mediation 'general pure gauge mediation' or pure GGM. This framework is broad enough to include everything from weakly coupled models with explicit messengers to strongly coupled theories with direct mediation.

Preliminary investigations of the phenomenology of GGM have been made in refs. [2-4]. In particular ref. [4] concentrated on the pure GGM scenario which we shall be adopting here. This is in a sense the most minimal assumption because it obviates the need for an additional sector just to generate the bilinear $B_{\mu}$ parameter for the higgses. To summarise the approach, in addition to the supersymmetric interaction,

$$
\mathcal{L}_{\text {eff }} \supset \int d^{2} \theta \mu \mathcal{H}_{u} \mathcal{H}_{d}
$$

the Higgs-sector effective Lagrangian also includes soft supersymmetry-breaking terms. All of the latter must be generated by the SUSY-breaking sector, since there would be little merit in a model of dynamical SUSY-breaking which generates only a subset of the SUSY-breaking terms in the effective SM Lagrangian. There are quadratic terms

$$
m_{u}^{2}\left|H_{u}\right|^{2}+m_{d}^{2}\left|H_{d}\right|^{2}+\left(B_{\mu} H_{u} H_{d}+\text { c.c. }\right),
$$

as well as cubic $a$-terms

$$
a_{u}^{i j} H_{u} Q^{i} \bar{u}^{j}+a_{d}^{i j} H_{d} Q^{i} \bar{d}^{j}+a_{L}^{i j} H_{d} L^{i} \bar{E}^{j},
$$


in the MSSM. As is well-known, a phenomenologically acceptable electroweak symmetry breaking in the supersymmetric SM occurs if $\mu^{2}$ and the soft masses in (1.2) at the low scale (i.e. the electroweak scale) are of the same order, $\mu^{2} \sim B_{\mu} \sim m_{\text {soft }}^{2} \sim M_{W}^{2}$.

In pure GGM we have no direct couplings of the SUSY-breaking sector to the Higgs sector, and therefore must have $B_{\mu} \approx 0$ at the messenger scale. From this starting point, i.e. taking $B_{\mu} \approx 0$ at the high scale $M_{\text {mess }}$, a small but viable value of $B_{\mu}$ is generated radiatively at the electroweak scale $[5,6]$. Electroweak symmetry breaking then determines the values of $\tan \beta$ and $\mu$. Since $B_{\mu}$ is small, $\tan \beta$ is generally large (between 20 and 70 ). This setup where $B_{\mu} \approx 0$ is an input and $\tan \beta$ is an output $[4,7]$ is in contrast to the common approach where $\tan \beta$ is taken as an arbitrary input and $B_{\mu}$ at the high scale is obtained from it.

The main free parameters are the gaugino and scalar masses as well as the messenger scale. For simplicity we restrict ourselves to a single effective scale $\Lambda_{G}$ for the gaugino masses and a single scale $\Lambda_{S}$ for the scalars. ${ }^{1}$ Thus at the messenger scale $M_{\text {mess }}$ the soft supersymmetry breaking gaugino masses are

$$
M_{\tilde{\lambda}_{i}}\left(M_{m e s s}\right)=k_{i} \frac{\alpha_{i}\left(M_{m e s s}\right)}{4 \pi} \Lambda_{G}
$$

where $k_{i}=(5 / 3,1,1), k_{i} \alpha_{i}$ (no sum) are equal at the GUT scale and $\alpha_{i}$ are the gauge coupling constants. The scalar mass squareds are

$$
m_{\tilde{f}}^{2}\left(M_{m e s s}\right)=2 \sum_{i=1}^{3} C_{i} k_{i} \frac{\alpha_{i}^{2}\left(M_{m e s s}\right)}{(4 \pi)^{2}} \Lambda_{S}^{2}
$$

where the $C_{i}$ are the quadratic Casimir operators of the gauge groups. Ordinary gauge mediation scenarios (see ref. [8] for a review) live on the restricted parameter space $\Lambda_{G} \simeq \Lambda_{S}$. We have implemented these boundary conditions in a modified version of Softsusy [9], which takes $\mu$ as an input and predicts $\tan \beta$ using the electroweak symmetry breaking conditions.

Outside the confines of ordinary gauge mediation the parameter space is populated by many models that predict different values of the ratio of gaugino to scalar masses, $\Lambda_{G} / \Lambda_{S}$. In models with explicit messengers one expects this ratio to be close to one, while for direct mediation models the gaugino masses are often suppressed relative to the scalar masses [7, 10-14]. Ref. [15] provided a general argument that linked the gaugino mass to the existence of lower lying minima at tree-level. Indeed hybrid models can easily be constructed which interpolate between these two cases by bringing lower lying minima in from infinity [16]. It is also possible to achieve values $\Lambda_{G} / \Lambda_{S}>1$ by increasing the "effective number of messengers" in the context of extraordinary gauge mediation models [17]. Naively this "gaugino mediation" region of parameter space corresponds to strong coupling, but explicit and calculable models space are possible in the context of extra dimensional models [18-23]

\footnotetext{
${ }^{1}$ Of course in each specific GGM model, the parameters $\Lambda_{G}$ and $\Lambda_{S}$ determining gaugino and scalar masses at the messenger scale are computed and expressed in terms of the scales of the SUSY-breaking sector, and details of the messenger fields. As such, $\Lambda_{G}$ and $\Lambda_{S}$ (together with $M_{\text {mess }}$ ) characterise a point in the pure GGM parameter space and can be treated as input parameters.
} 


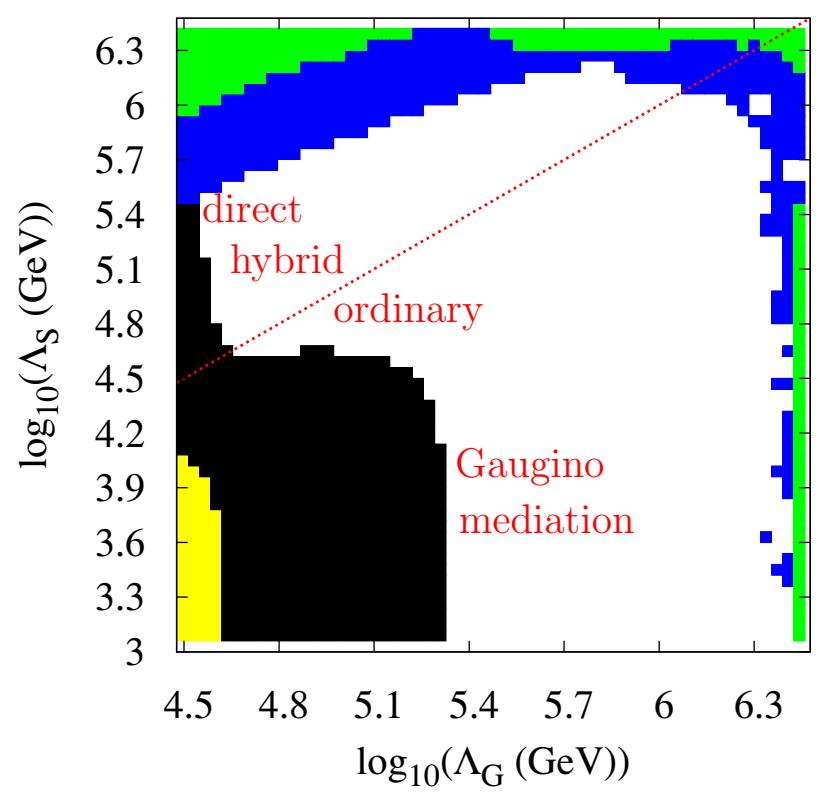

Figure 1. The underlying mediation physics corresponding to different regions of the $\Lambda_{G}, \Lambda_{S}$ parameter space. In the extreme $\Lambda_{G} \ll \Lambda_{S}$ region we have direct gauge mediation with no lower lying tree-level minima. Outside this region lies the hybrid region with lower lying minima being brought in from infinity. The red dotted line indicates the ordinary gauge mediation line where $\Lambda_{G}=\Lambda_{S}$, which can be reproduced in metastable set-ups with high messenger scales such as those in ref. [36]. Below the ordinary gauge mediation line we find the "many effective messenger" $\Lambda_{G} \gg \Lambda_{S}$ region, which is where some mechanism screens the contributions to the scalar masses. We also show the allowed region for intermediate messenger scales, $M_{\text {Mess }}=10^{10} \mathrm{GeV}$ with the dominant constraints excluding various areas indicated as follows: yellow (pale grey) means the point is excluded by the presence of tachyons in the spectrum, while the black region falls foul of the direct search limits. In the blue (dark grey) region SoftSUSY has not converged and in the green (light grey) region a coupling reaches a Landau pole during RG evolution.

or electric/magnetic duality [24] or some other mechanism which can screen the scalar mass contributions (see the latter reference for a more complete review). The broad relation of the underlying physics to the values of $\Lambda_{G}$ and $\Lambda_{S}$ is shown in figure 1 . It is striking that the phenomenology of GGM probes the vacuum structure so directly. We also show for later reference the exclusions from various phenomenological constraints discussed in detail in ref. [4] for a messenger scale of $10^{10} \mathrm{GeV}$. GGM allows also different $\Lambda_{G}^{(i)}$ and $\Lambda_{S}^{(j)}$ for the different species of gauginos and sfermions although certain sum-rules still apply [1]. However the general parameter space is prohibitively large for an exhaustive survey and moreover most perturbative models (for example the direct mediation models, or the hybrid models of [16]) do correspond to only to single $\Lambda_{G}, \Lambda_{S}$ and $M_{\text {mess }}$ scales. This is especially true if one wishes to maintain gauge coupling unification, which is most easily achieved by keeping an $\mathrm{SU}(5)$ structure for the mediating sector. In this sense the set of models defined by single $\Lambda_{G}, \Lambda_{S}$ and $M_{\text {mess }}$ scales are the gauge mediation equivalent of the canonical 
mSUGRA $^{2}$ scenario, with $\Lambda_{G}$ and $\Lambda_{S}$ playing the role of the parameters $m_{1 / 2}$ and $m_{0}$ in those models. ${ }^{3}$

The LHC is currently operating at $7 \mathrm{TeV}$ centre-of-mass energy and, it is hoped, will collect $1 \mathrm{fb}^{-1}$ of data by the end of 2011. It is thus relevant to ask what models and regions of parameter space might be discovered in the next year. Recent work on this subject includes [25-27] and has focussed on the mSUGRA scenario. The goal of this paper is to investigate the signatures and the discovery potential of pure GGM models at the early LHC stage focussing on collisions at $7 \mathrm{TeV}$. In section 2 we shall analyse the available parameter space relevant for this regime. We will proceed to construct a pair of benchmark points with relatively light gluinos. For these we compute the total $2 \rightarrow 2$ production cross-sections, the low-energy spectrum of superpartners and the branching ratios. The NLSP particles in this region of the parameter space are neutralinos.

We continue in section 3 with a more general survey of the NLSP phenomenology which is also very relevant for early stage LHC searches, and analyse other regions of the pure GGM parameter space, complementary to that of section 2. These will include benchmark points in the stau and co-NLSP regions, and a benchmark point in the $\Lambda_{G} \gg \Lambda_{S}$ region.

\section{Benchmark points with light gluinos for early LHC discovery}

We begin our investigation of the discovery potential of these models at the early LHC stage by focussing on two explicit benchmark points. The parameter space of pure GGM models was first investigated in our earlier work [4] which also excluded regions due to various constraints. These are shown for the example of a $10^{10} \mathrm{GeV}$ messenger scale in figure 1. We will be exploring the allowed regions of parameter space where either gluino or squark masses are likely to be sufficiently light to be discovered with a centre of mass energy of up to $7 \mathrm{TeV}$ and integrated luminosity of order $1 \mathrm{fb}^{-1}$. As a guideline note that in mSUGRA, ref. [26] has argued that when $m_{\tilde{g}} \sim m_{\tilde{q}}$ that the $1 \mathrm{fb}^{-1}$ reach is approximately 1.1 TeV . Our first two benchmark points will be chosen to have a slightly split spectrum with $m_{\tilde{q}} \sim 2-4 m_{\tilde{g}}$ to allow lighter gluinos.

Three scans of the parameter space of pure GGM are shown in figure 2, one at $M_{\text {mess }}=$ $10^{8} \mathrm{GeV}$ one at $M_{\text {mess }}=10^{10} \mathrm{GeV}$ and one at $M_{\text {mess }}=10^{14} \mathrm{GeV} \cdot{ }^{4}$ In each figure stop mass contours of $500 \mathrm{GeV}$ and $1 \mathrm{TeV}$ are indicated as dotted lines, and the $500 \mathrm{GeV}$ and $1 \mathrm{TeV}$ gluino contours are indicated as solid lines. ${ }^{5}$ Furthermore, the diagonal dotted red line corresponds to the boundary between neutralino and slepton NLSP. (Note that this line is similar to but distinct from the ordinary gauge mediation line of figure 1) The figures are also marked with a variety of benchmark points. The circular blobs are benchmark points

\footnotetext{
${ }^{2}$ We use the more common term minimal Supergravity (mSUGRA); Constrained MSSM (CMSSM) would be more accurate.

${ }^{3}$ Note that our approach is orthogonal to that taken in ref. [2] which has $\Lambda_{G}^{i}=\Lambda_{S}^{i}=\Lambda^{i}$, but a different $\Lambda^{i}$ for each gauge group.

${ }^{4}$ It should be noted that lower values of messenger scales restrict the parameter space significantly because of our assumption that $B_{\mu}$ is generated radiatively, and the fact that low messenger scales reduce the range of $\mathrm{RG}$ running.

${ }^{5}$ In the $M_{\text {mess }}=10^{8} \mathrm{GeV}$ scenario the single dotted contour is for $1 \mathrm{TeV}$ stop masses.
} 


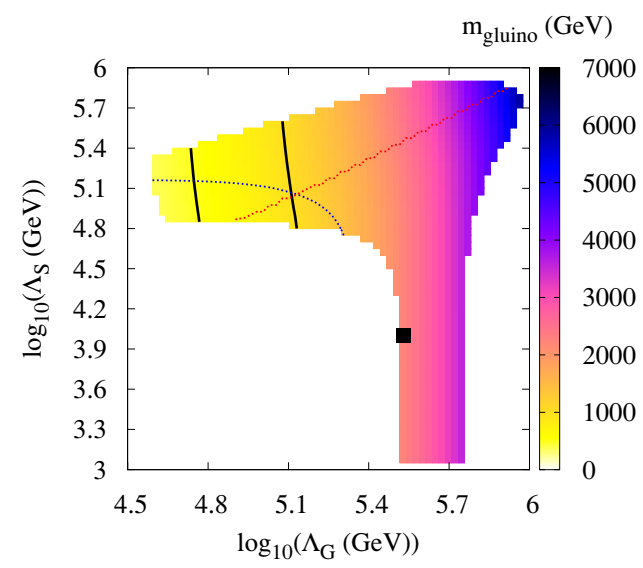

(a)

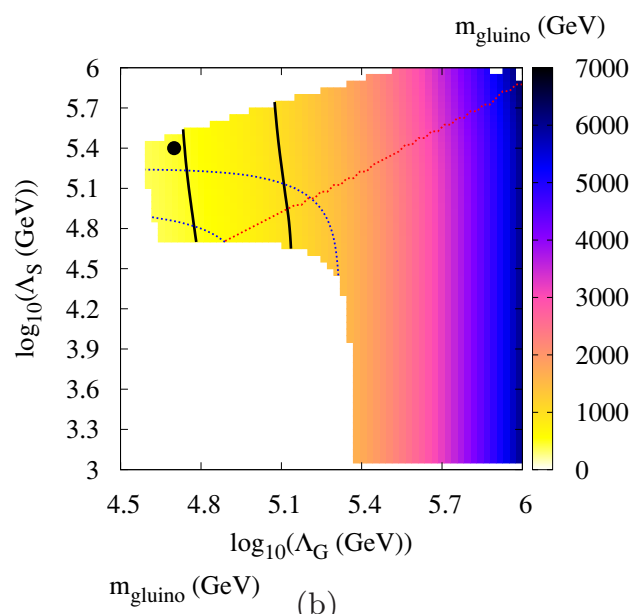

(b)

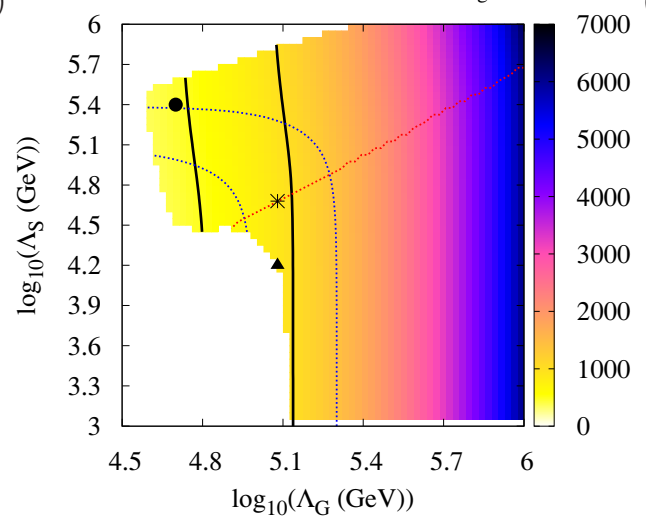

(c)

Figure 2. The $\Lambda_{G}, \Lambda_{S}$ parameter space for $M_{\text {mess }}=10^{8} \mathrm{GeV}$ (upper panel), $M_{\text {mess }}=10^{10} \mathrm{GeV}$ (middle panel) and $M_{\text {mess }}=10^{14} \mathrm{GeV}$ (lower panel). Stop mass contours $(500 \mathrm{GeV}$ and $1 \mathrm{TeV}$ ) are indicated as dotted lines, and the $500 \mathrm{GeV}$ and $1 \mathrm{TeV}$ gluino lines are solid. The NLSP is neutralino above the dotted red line and stau below. The marked points are the benchmark points discussed in the text: circular for neutralino NLSP (PGM1a middle panel, PGM1b bottom panel), triangular for stau NLSP (PGM2), a star for stau-neutralino co-NLSP (PGM3) on the bottom panel and finally a square for PGM4 which has stau NLSP and slepton NNLSP.

with a neutralino NLSP, the triangular points have a stau NLSP, and the stau-neutralino co-NLSP point is indicated by a star. The square blob corresponds to a gaugino mediated point with stau NLSP and slepton NNLSP. As will be seen from the $\chi^{2}$-analysis in figure 3 , the region where the squark masses are below $500 \mathrm{GeV}$ is somewhat disfavored by already existing data. Therefore, in this section we will concentrate on the region of the parameter space with light gluinos - benchmark points PGM1a and PGM1b. The triangular, square and star-shaped points with stau NLSP and stau-neutralino co-NLSP will be discussed in section 3.1.

We chose PGM1a and PGM1b in the light gluino region, to the left of the $500 \mathrm{GeV}$ line, marked as circular blobs in each figure. The first point (PGM1a) is for a medium to low messenger mass of $10^{10} \mathrm{GeV}$ (middle panel of figure 2). The second (PGM1b) is for a high messenger mass $10^{14} \mathrm{GeV}$ (lower panel of figure 2). These are typical light gluino 


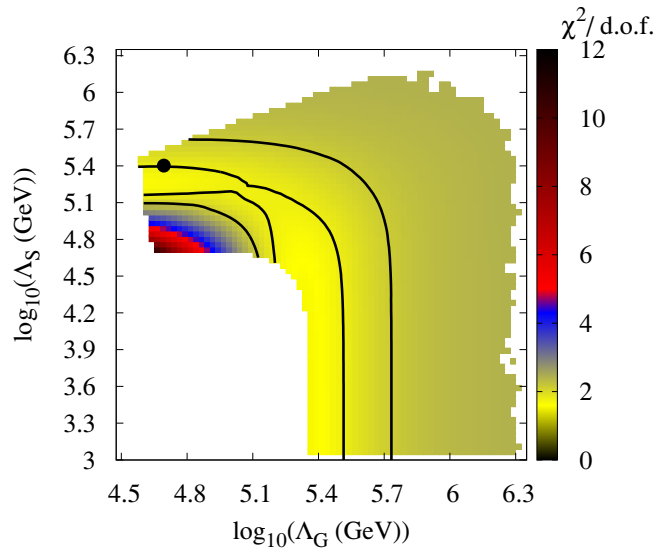

(a)

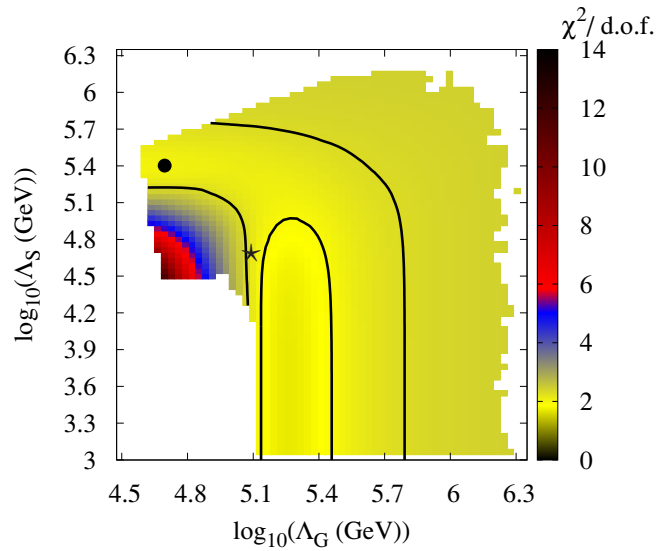

(b)

Figure 3. Figures $(\mathrm{a}, \mathrm{b})$ show the $\chi_{\text {tot }}^{2}$ distribution in the $\Lambda_{G}-\Lambda_{S}$ plane for $M_{m e s s}=10^{10}$ and $10^{14} \mathrm{GeV}$ respectively. The black lines denote the $68 \%$ and $95 \%$ confidence regions, and we also show the benchmark points following the same notation as before. The benchmark points are all inside the $95 \%$ confidence regions.

points, and as we have said correspond to phenomenology of the "mildly split" variety (in which the low energy spectrum is the Standard Model with only fermionic superpartners) found in the direct gauge mediation models analysed in refs. [7, 13, 14]. To some degree these points are quite generic: we chose them to be to the left of the $500 \mathrm{GeV}$ gluino line but we have not tried to optimize for the production cross section. The benchmark points are located in the regions of parameter space which are in good agreement with currently known experimental constraints. The experimental constraints were discussed in detail in ref. [4]. They are quantified by a total $\chi^{2}$ which it is pleasing to note is indeed low in these regions. As shown in figure 3, where the $68 \%$ and $95 \%$ confidence regions are indicated as black lines, both benchmark points lie well within the $95 \%$ confidence region.

The spectra of the two benchmark points are given in table 1, and the neighbourhood of the chosen benchmark points leads to similar spectra. The main features of the spectrum in these points are that they have light gluinos with masses below $500 \mathrm{GeV}$ and that the NLSP is a bino-like neutralino. ${ }^{6}$ Detailed discussion of other possibilities for NLSP phenomenology in the early stages of the LHC in PGGM will be presented in section 3.

For the rest of the spectrum in table 1 we note that the first two neutralinos are light, while the Higgsino-like third and fourth neutralinos are much heavier, at the TeV scale. A similar story holds for the charginos: one is quite light, approximately $135 \mathrm{GeV}$ and is wino-like while the other is higgsino-like and at the TeV scale. The left-handed sleptons are at the $\mathrm{TeV}$ scale, while the right-handed ones vary from $400-700 \mathrm{GeV}$ depending on the point and sparticle type. As usual, the right-handed staus are the lightest of the sleptons due to mixing proportional to $\tan \beta$ and the relatively large size of $\lambda_{\tau}$. Finally, the squarks all have masses above $1 \mathrm{TeV}$. Thus for these benchmark points the dominant production channel at the LHC is gluino pair production.

\footnotetext{
${ }^{6}$ The LSP is the gravitino as is standard in gauge mediation.
} 


\begin{tabular}{|c|c|c|}
\hline Benchmark point & PGM1a & PGM1b \\
\hline$M_{\text {mess }}(\mathrm{GeV})$ & $10^{10}$ & $10^{14}$ \\
\hline$\Lambda_{G}(\mathrm{GeV})$ & $5 \times 10^{4}$ & $5 \times 10^{4}$ \\
\hline$\Lambda_{S}(\mathrm{GeV})$ & $2.5 \times 10^{5}$ & $2.5 \times 10^{5}$ \\
\hline $\tan \beta$ & 46.6 & 41.2 \\
\hline$\chi_{1}^{0}$ & 67 & 67 \\
\hline$\chi_{2}^{0}$ & 136 & 133 \\
\hline$\chi_{3}^{0}$ & 1038 & 936 \\
\hline$\chi_{4}^{0}$ & 1039 & 938 \\
\hline$\chi_{1}^{ \pm}$ & 136 & 134 \\
\hline$\chi_{2}^{ \pm}$ & 1039 & 937 \\
\hline$\tilde{g}$ & 458 & 453 \\
\hline$\tilde{e}_{L}, \tilde{\mu}_{L}$ & 927 & 1013 \\
\hline$\tilde{e}_{R}, \tilde{\mu}_{R}$ & 540 & 712 \\
\hline$\tilde{\tau}_{1}$ & 392 & 544 \\
\hline$\tilde{\tau}_{2}$ & 898 & 964 \\
\hline$\tilde{\nu}_{1,2}$ & 925 & 1011 \\
\hline$\tilde{\nu}_{3}$ & 889 & 958 \\
\hline$\tilde{t}_{1}$ & 1418 & 1050 \\
\hline$\tilde{t}_{2}$ & 1729 & 1471 \\
\hline$\tilde{b}_{1}$ & 1578 & 1287 \\
\hline$\tilde{b}_{2}$ & 1731 & 1471 \\
\hline$\tilde{u}_{L}, \tilde{c}_{L}$ & 2011 & 1760 \\
\hline$\tilde{u}_{R}, \tilde{c}_{R}$ & 1803 & 1520 \\
\hline$\tilde{d}_{L}, \tilde{s}_{L}$ & 1983 & 1734 \\
\hline$\tilde{d}_{R}, \tilde{s}_{R}$ & 1774 & 1460 \\
\hline$h_{0}$ & 116.9 & 115.3 \\
\hline$A_{0}, H_{0}$ & 944 & 1032 \\
\hline$H^{ \pm}$ & 947 & 1035 \\
\hline
\end{tabular}

Table 1. Spectra for the two benchmark points with light gluinos. All masses are in GeV. The NLSP and the lightest coloured super-particle (gluino) are shown in bold in each case. These spectra and all other relevant details can be obtained in SLHA format at http://www.ippp.dur.ac.uk/ SUSY.

We have computed the total production cross-sections to NLO using PROSPINO [28, 29]. The total gluino production cross sections in pp collissions at $7 \mathrm{TeV}$ are,

$$
\begin{array}{lll}
\text { PGM1a : } & \sigma_{p p \rightarrow \tilde{g} \tilde{g}}=4.09 \mathrm{pb} & @ 7 \mathrm{TeV} \\
\text { PGM1b : } & \sigma_{p p \rightarrow \tilde{g} \tilde{g}}=4.34 \mathrm{pb} & @ 7 \mathrm{TeV}
\end{array}
$$

We present cross-sections in femtobarns for various channels in table 2. Since before shutdown the early-stage LHC is expected to accumulate approximately $1 \mathrm{fb}^{-1}$ of luminosity, the entries in the table also give the number of SUSY events expected before then. The 


\begin{tabular}{|c|c|c|c|c|}
\hline Benchmark Point & $\sigma_{p p \rightarrow \tilde{g} \tilde{g}}$ & $\sigma_{p p \rightarrow \chi_{2}^{0} \chi_{1}^{ \pm}}$ & $\sigma_{p p \rightarrow \chi_{1}^{+} \chi_{1}^{-}}$ & $\sigma_{p p \rightarrow \tilde{g} \tilde{q}}$ \\
\hline PGM1a & 4090 & 2682 & 1320 & 18.9 \\
\hline PGM1b & 4340 & 2835 & 1390 & 58.7 \\
\hline
\end{tabular}

Table 2. Production rates for the most important processes for the two benchmark points under consideration at the LHC with $\sqrt{s}=7 \mathrm{TeV}$. All cross-sections are in femtobarns.

largest contribution to the total production cross-section comes from gluino production, as the gluinos are both relatively light and strongly interacting. Since for our benchmark points the sfermions are significantly heavier than the gauginos, production processes involving the squarks are suppressed relative to those only involving gluinos.

Weak gaugino pair production also makes a large contribution to the total cross-section. Since $\chi_{2}^{0}$ is wino-like, the cross-sections for $\chi_{2}^{0} \chi_{1}^{ \pm}$production are much higher than for the same process with $\chi_{2}^{0}$ replaced with the bino-like $\chi_{1}^{0}$. Di-chargino production, with a cross-section of 1.32 (1.39) pb for PGM1a (PGM1b, respectively) also makes an important contribution. All other cross-sections are nearly two orders of magnitude smaller than these, such as $p p \rightarrow \tilde{g} \tilde{q}$, also shown in table 2 . We have also investigated all the other possibilities, $p p \rightarrow \chi_{i}^{0} \chi_{j}^{ \pm}$, in this family of processes. The Higgsino nature of $\chi_{3,4}^{0}$ and $\chi_{2}^{ \pm}$ means that production of these particles is negligible. Even though the lightest neutralino has $m_{\chi_{1}^{0}}=67 \mathrm{GeV}$, it is not directly produced in any great numbers. Of course, these features will change in regions where the hierarchy between the sfermions and gauginos is less pronounced, and also when the centre of mass energy is raised from 7 to $14 \mathrm{TeV}$. The decays of the lightest chargino are dominated by $\chi_{1}^{+} \rightarrow \chi_{1}^{0} q_{u} \bar{q}_{d}$, which occurs $69 \%$ $(70 \%)$ of the time. The rest of the branching ratio is taken up by $\chi_{1}^{+} \rightarrow l^{+} \nu_{l}$, where $l=(e, \mu, \tau)$, with the tau-component taking a somewhat larger share of $19 \%(20 \%)$. The wino-like neutralino $\chi_{2}^{0}$ decays predominantly to $\chi_{1}^{0} q \bar{q}, 71 \%$ (87\%), and to $\chi_{1}^{0} \tau^{+} \tau^{-}, 23 \%$ (5\%), with the remaining channels being a combination of $\chi_{1}^{0} l_{e, \mu}^{+} l_{e, \mu}^{-}$and $\chi_{1}^{0} \nu \bar{\nu}$. The upshot of this analysis is that in both $\chi_{1}^{+} \chi_{1}^{-}$and $\chi_{1}^{ \pm} \chi_{1}^{0}$ production the standard $4 j+$ MET analysis should be useful for probing supersymmetry this year.

One might wonder about the existing strong constraints on NLSP neutralino and chargino masses from Tevatron (see [30] for an overview). The strongest constraints resulting in a high lower bound on neutralino and chargino masses originate from two potential signals. The first one is a di-photon signature studied most recently in refs. [31, 32]. In this case one considers production and subsequent decay $p \bar{p} \rightarrow \chi_{1}^{+} \chi_{1}^{-} \rightarrow 2 \chi_{1}^{0}+\ldots \rightarrow 2 \gamma+2 \tilde{G}+\ldots$ or $p \bar{p} \rightarrow \chi_{2}^{0} \chi_{1}^{ \pm} \rightarrow 2 \chi_{1}^{0}+\ldots \rightarrow 2 \gamma+2 \tilde{G}+\ldots$. The (unobserved) signal is two photons plus missing transverse energy. However, for such bounds to hold the last decay stage of a neutralino NLSP into a photon and a gravitino must happen promptly (at the very least inside the detector). In general prompt NLSP decays occur only for sufficiently low messenger masses. As we will see in more detail in section 3 (see figure 6) the NLSP decays happen way outside the detector for our benchmark points PGM1a,b.

The second signature analysed at Tevatron is a tri-lepton signal. The production would follow from $p \bar{p} \rightarrow \chi_{2}^{0} \chi_{1}^{ \pm} \rightarrow 2 \chi_{1}^{0}+\ell \bar{\ell}+\ell^{\prime} \nu$. This signal has been analysed in refs. [33, 34] in the context of mSugra with low values of $\tan (\beta)=3$, setting a new lower limit on chargino 

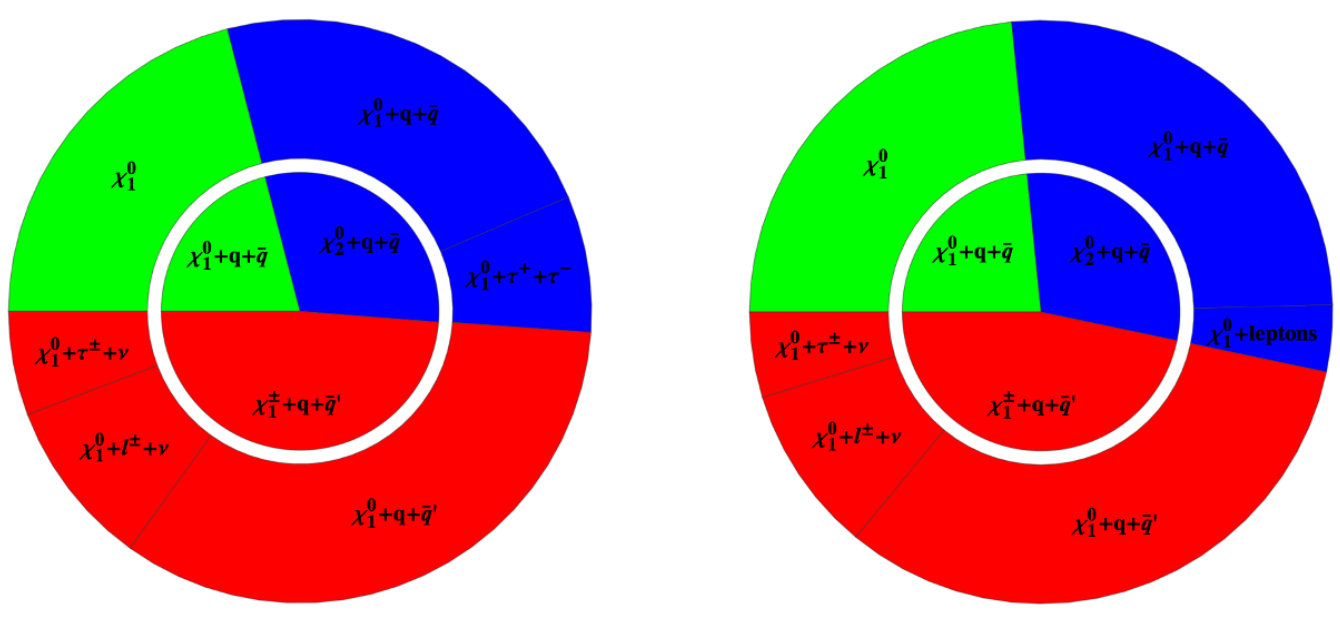

Figure 4. Piecharts giving a rough impression of the gluino decay chains/branching ratios with the PGM1a benchmark point on the left panel and PGM1b on the right. In the first step the gluino decays into the products depicted in the inner ring, in the next step the daughter sparticle decays into the products given in the outer ring (for simplicity we only write down the additional decay products for this last decay). We do not display those chains with a branching ratio less than $5 \%$.

masses of $164 \mathrm{GeV}$. However, the value of the upper limit depends quite strongly on the choice made for $\tan (\beta)$ as well as other model dependent considerations. Therefore it will be different in gauge mediation. In particular all our predictions obtained in a pure GGM setup always have much higher values of $\tan (\beta)$. This increases the branching fraction to $\tau \mathrm{s}$ which are more difficult to reconstruct. Overall the branching ratios to leptons are quite small in our scenarios as can be seen from the red and blue segments of the outer circles in figure 4. This makes the current constraints inconclusive for the pure GGM predictions analyzed here. This, of course, can be changed by an analysis of (existing) larger sets of Tevatron data, which would be very interesting. We now focus on $p p \rightarrow \tilde{g} \tilde{g}$, and discuss the main decay avenues to the final states including NLSPs. It can be seen from this analysis that the gluino decays dominantly into a chargino plus a quark and an antiquark. Subsequently the chargino decays into a neutralino plus either a quark and an antiquark, or a lepton and a neutrino, as discussed above. An alternative interesting channel is that each gluino decays directly into a neutralino and a quark-antiquark pair. In all of these processes the two gluinos will decay into a total of 4 or more coloured particles and two neutralinos (plus leptons in some cases). In figure 4 the branching ratios of the gluino and the daughter sparticle decays are represented graphically, with the PGM1a benchmark point shown on the left panel and the PGM1b point on the right. Decay chains with branching ratios of less than $5 \%$ are not shown. ${ }^{7}$ The full set of branching ratios (as well as the spectra in

\footnotetext{
${ }^{7}$ For example, for the PGM1a the $\chi_{2}^{0}$ decays to $23 \%$ into $\tau$ 's but only to less than $4 \%$ into other leptons and thus the latter are not shown on the left panel in figure 4. For the PGM1b the $\chi_{2}^{0}$ decays $5 \%$ into $\tau$ 's and to nearly $4 \%$ into other leptons, these two contributions are combined and collectively called "leptons" on the right panel in figure 4.
} 
SLHA format) for these benchmark points can be found at

$$
\text { http://www.ippp.dur.ac.uk/ SUSY }
$$

In the following section we will present a more general overview of the NLSP phenomenology. We shall then perform a complementary analysis, in regions of the parameter space where the NLSP is a stau or a light slepton or there are co-NLSPs (in practice these are areas where the stau and neutralino are nearly degenerate in mass). Again we focus on areas that may be relevant to the early LHC searches.

\section{Survey of NLSP phenomenology}

In gauge mediated models the Lightest Supersymmetric Particle (LSP) is always the gravitino [8]. There is much interest therefore in the phenomenology of the Next-to-LSP (NLSP) as this is the metastable state into which any produced superpartner will decay before ultimately decaying to the gravitino. Therefore it is instructive to map out the NLSP phenomenology in the whole $\Lambda_{G}, \Lambda_{S}$ parameter space, and describe in more detail some of the top-down models that correspond to the different regions.

For the assumptions we outlined above, the NLSP is either slepton or neutralino. The NLSP phenomenology is of great interest for two reasons [8]. First it is typically very long lived - its decay to the gravitino is suppressed: $\Gamma \propto m_{N L S P}^{5} / F_{0}^{2}$ where $m_{N L S P}$ is its mass and $F_{0}$ is the intrinsic scale of supersymmetry breaking in the hidden sector (i.e. the potential is $\langle V\rangle=F_{0}^{2}$ ). Typically, depending on how the SUSY breaking encoded by $F_{0}$ is mediated, $\Gamma$ represents many orders of magnitude of suppression. If it is sufficiently long lived the NLSP will exit the detector as missing energy, or leave a muon-like track if it is charged (e.g. if it is a stau). On the other hand for certain values of parameters (which we discuss presently) the particle can decay inside the detector possibly allowing one to resolve a displaced decay vertex. Moreover such a measurement would give direct information about the SUSY breaking in the hidden sector $F_{0}$ rather than that seen in the visible sector which depends heavily on the particular type of (gauge) mediation. The compositions of the NLSP in different regions of parameter space are shown in figure 5, again one at $M_{\text {mess }}=10^{8} \mathrm{GeV}$ (top), one at $M_{\text {mess }}=10^{10} \mathrm{GeV}$ (middle) and one at $M_{\text {mess }}=10^{14} \mathrm{GeV}$ (bottom). In each figure again stop mass contours of $500 \mathrm{GeV}$ and $1 \mathrm{TeV}$ are indicated as dotted lines, and the $500 \mathrm{GeV}$ gluino contour is indicated as a solid line. We have indicated 3 different NLSP regions on the figures, each giving quite distinct experimental signatures:

- Neutralino NLSP (Marked in green): no ionization track and either missing energy or displaced vertex with decay predominantly to photon $\left(\chi_{1}^{0} \rightarrow \tilde{G} \gamma\right)$ or jet/lepton pairs $\left(\chi_{1}^{0} \rightarrow \tilde{G} Z \rightarrow \tilde{G}+j e t s / l \bar{l}\right)$.

- Stau NLSP (Marked in blue): ionization track plus possible displaced vertex with decay predominantly to jets $\left(\tilde{\tau}_{R} \rightarrow \tilde{G} \tau \rightarrow \tilde{G} \nu_{\tau}+j e t s / l^{\prime} \bar{l}\right)$.

- Neutralino/stau co-NLSP (Marked in red): if the mass difference between the neutralino and stau is less than $m_{\tau}$, then the NNLSP is unable to decay to the NLSP, 


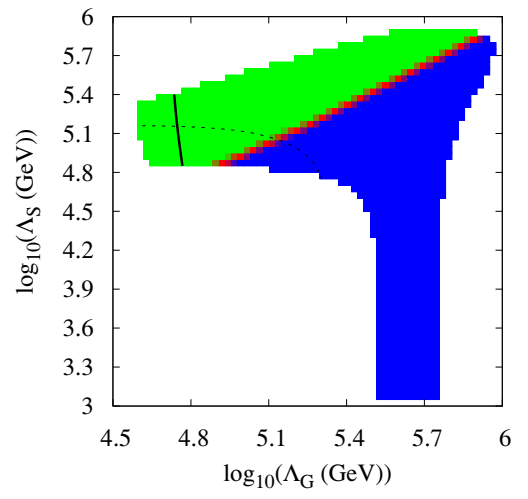

(a)

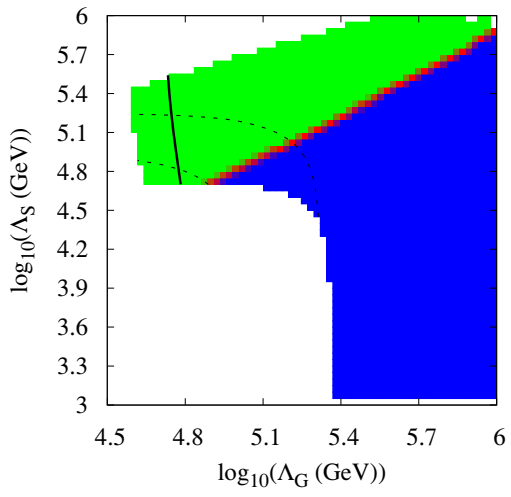

(b)

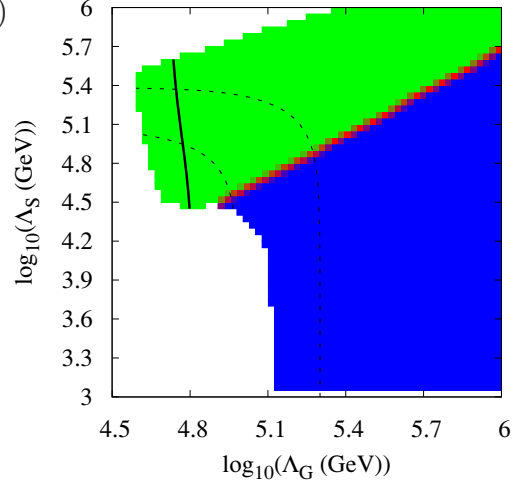

(c)

Figure 5. The NLSP regions in the $\Lambda_{G}, \Lambda_{S}$ parameter space for $M_{\text {mess }}=10^{8} \mathrm{GeV}$ (top figure), $M_{\text {mess }}=10^{10} \mathrm{GeV}$ (middle figure) and $M_{\text {mess }}=10^{14} \mathrm{GeV}$ (bottom figure). The NLSP is $\chi_{1}^{0}$ in the green region, $\chi_{1}^{0} / \tilde{\tau}$ co-NLSP in the red region and $\tilde{\tau}$ in the blue region.

and each component behaves effectively a separate NLSP. One expects a mix of those previous two cases.

We can treat the decay length of the NLSP as follows. First consider the decays: they go through the interaction term which for on-shell particles is [8]

$$
\mathcal{L}=\frac{1}{F_{0}}\left(\left(m_{f}^{2}-m_{\tilde{f}}^{2}\right) \bar{f}_{L} \tilde{f}+\frac{M_{\tilde{\lambda}_{i}}}{4 \sqrt{2}} \overline{\tilde{\lambda}}_{i} \sigma^{\mu \nu} F_{\mu \nu}^{i}\right) \tilde{G}+\text { h.c. }
$$

where $\tilde{G}$ is the Goldstino and as we have already stated $F_{0}$ is the absolute scale of supersymmetry breaking. The decay length derived from eq. (3.1) is given by

$$
L_{\text {decay }}=\frac{1}{\kappa}\left(\frac{100 \mathrm{GeV}}{m_{N L S P}}\right)^{5}\left(\frac{F_{0}}{(100 \mathrm{TeV})^{2}}\right)^{2} 0.1 \mathrm{~mm}
$$

where the factor $\kappa$ is a calculable number depending on the mixing in the NLSP, and is of order unity (precisely unity for the stau in fact). The interesting case is when decay takes place inside the detector which conservatively requires $L_{\text {decay }}<10 \mathrm{~m}$. For NLSP masses less that $500 \mathrm{GeV}$, this translates into

$$
\sqrt{F_{0}} \lesssim 10^{4} \mathrm{TeV} .
$$


Thus $F_{0}$ will be at the lower end of the possible range.

In order to get more precise information we need to consider the relation between $F_{0}$ and $\Lambda_{G}$ or $\Lambda_{S}$. This is very model dependent, but simplifies if we take there to be only one source of supersymmetry breaking (i.e. one potential Goldstino) and one dominant source of mediation for gauginos or scalars. Under this assumption the relation between the $\Lambda$ 's and $F_{0}$ can be expressed with two parameters $k_{G}$ and $k_{S}$ as

$$
\Lambda_{G}=k_{G} F_{0} / M_{m e s s} ; \Lambda_{S}=k_{S} F_{0} / M_{m e s s} .
$$

In GGM, $k_{G}$ and $k_{S}$ are independent parameters which encode the difference between the gauge and scalar mass scales $\Lambda_{G}$ and $\Lambda_{S}$. In ordinary gauge mediation, $k_{G}=k_{S}$, and this corresponds to a simple one-scale special case of GGM. In general, as will be reviewed shortly, the range of values for $k_{G}$ and $k_{S}$ is highly model-dependent.

In order to present model-independent information it is useful to express $F_{0}$ with reference to $\Lambda_{G}$ : i.e. we replace $F_{0}=k_{G}^{-1} \Lambda_{G} M_{\text {mess }}$. The decay length $L_{\text {decay }}$ derived from eq. (3.1) is given by

$$
k_{G}^{2} L_{\text {decay }}=\frac{1}{\kappa}\left(\frac{100 \mathrm{GeV}}{m_{N L S P}}\right)^{5}\left(\frac{\sqrt{\Lambda_{G} M_{\text {mess }}}}{100 \mathrm{TeV}}\right)^{4} 0.1 \mathrm{~mm}
$$

We then plot contours of $k_{G}^{2} L$. The reason that this is a most useful parameterization is that in the regions where $\Lambda_{G}>\Lambda_{S}$ the NLSP is mainly slepton, as can be seen from figure 5 , and its mass is dominated by renormalization group contributions from the gauginos (except when $\Lambda_{G} / \Lambda_{S} \sim \mathcal{O}(1-10)$ ). Thus $m_{N L S P}$ is mainly a function of $\Lambda_{G}$ (just as the stop mass is in fact). On the other hand in the regions where $\Lambda_{G}<\Lambda_{S}$ the NLSP is mainly a bino-like neutralino and again its mass is expected to be dominated by $\Lambda_{G}$. Hence the r.h.s. of eq. (3.5) is predominantly a function of $\Lambda_{G}$.

We show the results for the decay lengths $\log _{10}\left(k_{G}^{2} L_{\text {decay }}\right)$ in figure 6 for the three values of the messenger mass. We see that the contours follow a vertical, horizontal and vertical again pattern, which we now explain. Starting at the top of the figures, when $\Lambda_{S}$ is large the NLSP is the neutralino, and the decay length does not change with decreasing $\Lambda_{S}$ as both $m_{N L S P}$ and $\Lambda_{G}$ are constant. When the NLSP species changes from neutralino to the lightest stau, there is a kink in the contour. This is partly due to the change in $\kappa$, and also to the change in the behaviour of the NLSP mass with $\Lambda_{G}$ and $\Lambda_{S}$. In this regime the stau mass is dominated by $\Lambda_{S}$ and, although $k_{G}^{2} L_{\text {decay }}$ is proportional to $\Lambda_{G}^{2}$ the factor of $1 / m_{\tilde{\tau}}^{5}$ means that $k_{G}^{2} L_{\text {decay }}$ is proportional to $1 / \Lambda_{S}^{5}$. When these two parameters are of the same of order of magnitude the contour thus appears flat in $\Lambda_{S}$. Finally, when $\Lambda_{G} / \Lambda_{S} \sim 10$ the stau mass begins to be dominated by $\Lambda_{G}$ and generated mostly through RG running and so the contour is again approximated by a line of constant $\Lambda_{G}$.

It is instructive to now consider the values of $k_{G}$ that one expects to have in various different top-down scenarios in order to see whether decays inside the detector are a possibility:

- Ordinary mediation: Here one has only one messenger and $\Lambda_{G}=\Lambda_{S}$ and $k_{G}$ is the coupling of the messenger to the SUSY breaking $F$-term. Typically one takes $k_{G} \sim 1$. 


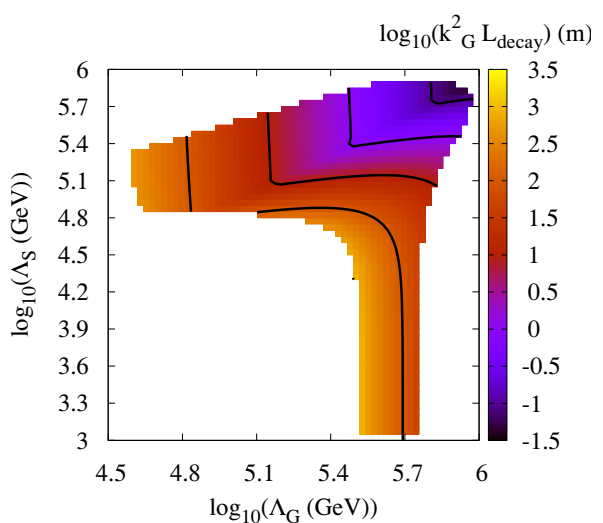

(a)

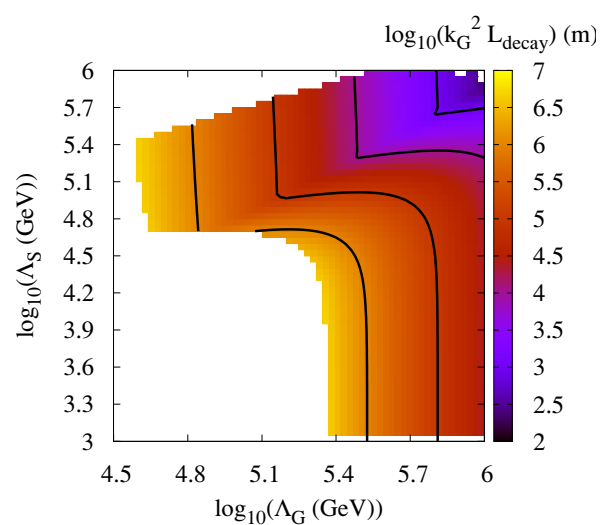

$\log _{10}\left(\mathrm{k}_{\mathrm{G}}{ }^{2} \mathrm{~L}_{\text {decay }}\right)(\mathrm{B})$

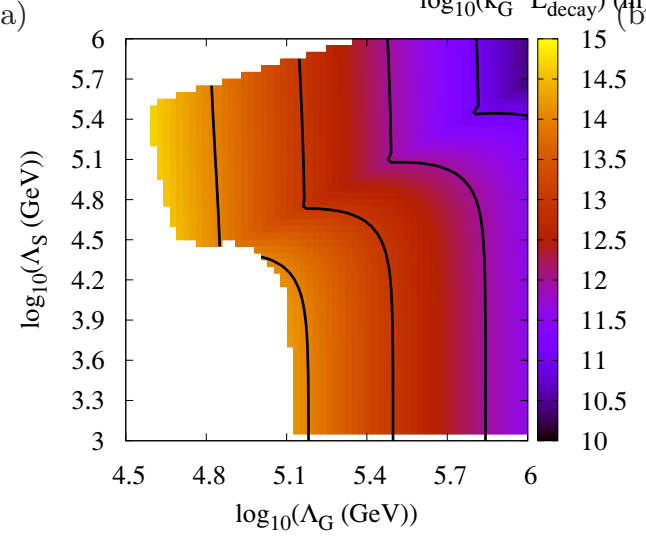

(c)

Figure 6. This figure shows the logarithm of the decay length in meters of the NLSP, $\log _{10}\left(k_{G}^{2} L_{\text {decay }}\right)$ for $M_{\text {mess }}=1 \times 10^{8} \mathrm{GeV}$ (top), $M_{\text {mess }}=1 \times 10^{10} \mathrm{GeV}$ (middle) and $M_{\text {mess }}=$ $1 \times 10^{14} \mathrm{GeV}$ (bottom), as well as contours for each case.

In this case figure 6 gives directly the decay lengths of the NLSP. Evidently low messenger scales are required for decay inside the detector. For $M_{\text {mess }}=10^{8} \mathrm{GeV}$ decays can happen inside or outside the detector, depending on the region of parameter space. Comparing figure 6 with figure 2 we see that decay inside the detector happens when $m_{\tilde{g}} \geq 1 \mathrm{TeV}$. Intermediate scales $M_{\text {mess }}=10^{10} \mathrm{GeV}$ would require high values of $\Lambda_{G}, \Lambda_{S}$ which leads to very high masses outside the early discovery region.

- Suppressed ordinary gauge mediation: ref. [36] presented a simple scheme for gauge mediation in which a single messenger field was coupled to a metastable SUSYbreaking sector of the type introduced in ref. [37]. In these models the Goldstino superfield is a composite particle (a "meson") and hence the effective coupling to the messenger fields is suppressed by a factor $k_{G} \sim k_{S} \sim \frac{\Lambda_{c o m p}}{M_{X}} \ll 1$ where $M_{X}$ is some high fundamental scale which might be $M_{\mathrm{Pl}}$, and $\Lambda_{\text {comp }}$ is the scale of compositeness. The general expectation is that $k_{G}, k_{S} \ll 1$ and indeed phenomenological viability demands it. For example the values chosen in ref. [36] give $k_{G}, k_{S} \sim 10^{-7}$. Hence decay inside the detector (or indeed the Solar system) is clearly impossible for any values of $M_{\text {mess }}$ or $\Lambda_{G}, \Lambda_{S}$. 
- Mildly split spectrum: phenomenology of the "mildly split" variety (in which the low energy spectrum is the Standard Model plus only the fermionic superpartners) was found in the direct gauge mediation models analysed in refs. [7, 13, 14]. This type of phenomenology is in fact characteristic of models that have no tree-level metastability, due to a theorem by Komargodski and Shih [15] that tree-level gaugino masses are equivalent to there existing some point in moduli space where there is a tachyon. These models have $\Lambda_{G} \ll \Lambda_{S} \lesssim F_{0}$ and hence they correspond to $k_{S} \sim 1$ and $k_{G} \sim 10^{-(1-2)}$. Since the NLSP mass is governed by $\Lambda_{G}$, viable phenomenology requires larger values of $F_{0}$ and a commensurately slower NSLP decay. For example low messenger scales $M_{\text {mess }}=10^{8} \mathrm{GeV}$ can just give decay within the detector whereas already intermediate scales $M_{\text {mess }}=10^{10} \mathrm{GeV}$ do not allow decay within the detector at all.

- Many messenger/strong coupling limit: In ordinary gauge mediation, the gaugino mass scale $\Lambda_{G}$ is proportional to the number of messengers $N_{\text {mess }}$, and the mediated squark mass scale $\Lambda_{S}$ is proportional to $\sqrt{N_{\text {mess }}}$. Thus in the "many effective messengers" limit, we access the $\Lambda_{G} \gg \Lambda_{S}$ region of the parameter space and moreover we can effectively have $k_{G} \gg 1$, so the NLSP decays more rapidly. For example when $k_{G} \sim 30$ even intermediate messenger masses, $M_{\text {mess }}=10^{10} \mathrm{GeV}$, allow NLSP decays to take within the detector with reasonably low masses for the coloured sparticles (i.e. below $1 \mathrm{TeV}$ ). Of course naively adding many messengers leads to strong coupling in the visible sector: as discussed in the introduction calculable models in this region require some mechanism to screen the scalar mass contribution.

To summarise the discussion arising from figure 6: in most cases NLSP decay happens well outside the detector. Decays inside the detector are only possible for relatively low messenger masses, high SUSY breaking scales and/or quite strong coupling $k_{G}$ to the hidden sector.

\subsection{Stau and co-NLSP Benchmark points}

As can be seen from figure 5 , the stau NLSP and co-NLSP regions both have $m_{\tilde{g}}>500 \mathrm{GeV}$ and $m_{\tilde{q}}>500 \mathrm{GeV}$. The low-mass parts of these regions are also disfavoured according to the analysis of supersymmetric contributions to Standard Model observables in [4]. Accordingly the production cross-sections in these cases are lower than for neutralino NLSP. However, in the stau NLSP case (and also possibly in the co-NLSP scenario) with higher messenger scales the stau is stable on collider length- and time-scales. The signatures from such charged massive metastable particles (CHAMPS) are unique enough that early SUSY discovery may be feasible even with the smaller cross-sections in this scenario. We have therefore selected two benchmark points, PGM2 with a stau NLSP, and PGM3 with a stau co-NLSP (both shown on the bottom panel in figure 2) and performed a preliminary analysis of their phenomenology. In addition we have for completeness chosen a fifth benchmark point, PGM4, at low messenger scales in the $\Lambda_{G} \gg \Lambda_{S}$ region (with $\Lambda_{G}=3.4 \times 10^{5} \mathrm{GeV}$ and $\left.\Lambda_{G}=10^{4} \mathrm{GeV}\right)$. As for the light gluino points, the SLHA files and are available on the Pure GGM website mentioned above. The spectra are shown in table 3. 


\begin{tabular}{|c|c|c|c|}
\hline Benchmark point & PGM2 & PGM3 & PGM4 \\
\hline$M_{\text {mess }}(\mathrm{GeV})$ & $10^{14}$ & $10^{14}$ & $10^{8}$ \\
\hline$\Lambda_{G}(\mathrm{GeV})$ & $1.2 \times 10^{5}$ & $1.2 \times 10^{5}$ & $3.4 \times 10^{5}$ \\
\hline$\Lambda_{S}(\mathrm{GeV})$ & $1.6 \times 10^{4}$ & $4.76 \times 10^{4}$ & $10^{4}$ \\
\hline $\tan \beta$ & 19.0 & 20.5 & 34.4 \\
\hline$\chi_{1}^{0}$ & 156 & 157 & 456 \\
\hline$\chi_{2}^{0}$ & 292 & 296 & 723 \\
\hline$\chi_{3}^{0}$ & 461 & 489 & 743 \\
\hline$\chi_{4}^{0}$ & 479 & 504 & 897 \\
\hline$\chi_{1}^{ \pm}$ & 291 & 295 & 720 \\
\hline$\chi_{2}^{ \pm}$ & 480 & 505 & 898 \\
\hline$\tilde{g}$ & 879 & 887 & 2239 \\
\hline$\tilde{e}_{L}, \tilde{\mu}_{L}$ & 246 & 305 & 406 \\
\hline$\tilde{e}_{R}, \tilde{\mu}_{R}$ & 129 & 182 & 163 \\
\hline$\tilde{\tau}_{1}$ & 100 & 157 & 110 \\
\hline$\tilde{\tau}_{2}$ & 254 & 310 & 423 \\
\hline$\tilde{\nu}_{1,2}$ & 234 & 296 & 401 \\
\hline$\tilde{\nu}_{3}$ & 232 & 293 & 401 \\
\hline$\tilde{t}_{1}$ & 618 & 650 & 1459 \\
\hline$\tilde{t}_{2}$ & 786 & 823 & 1601 \\
\hline$\tilde{b}_{1}$ & 726 & 769 & 1557 \\
\hline$\tilde{b}_{2}$ & 761 & 802 & 1596 \\
\hline$\tilde{u}_{L}, \tilde{c}_{L}$ & 804 & 860 & 1682 \\
\hline$\tilde{u}_{R}, \tilde{c}_{R}$ & 766 & 810 & 1621 \\
\hline$\tilde{d}_{L}, \tilde{s}_{L}$ & 795 & 850 & 1658 \\
\hline$\tilde{d}_{R}, \tilde{s}_{R}$ & 765 & 805 & 1621 \\
\hline$h_{0}$ & 113.3 & 113.4 & 118 \\
\hline$A_{0}, H_{0}$ & 493 & 539 & 781 \\
\hline$H^{ \pm}$ & 499 & 545 & 785 \\
\hline
\end{tabular}

Table 3. Spectra for three benchmark points with stau NLSP. PGM2 has slepton NNLSP and a high messenger scale and PGM3 has stau-neutralino co-NLSP also at a high messenger scale. PGM4 is at low messenger scale with slepton NNLSP. All masses are in GeV. The NLSP is shown in bold in each case. These spectra and all other relevant details can be obtained in SLHA format at http://www.ippp.dur.ac.uk/ SUSY.

Let us first consider the stau NLSP case, PGM2. Due to the constraint from the Higgs mass, it is not possible to have very light squarks in this case. The point we have chosen has $\Lambda_{G}=1.2 \times 10^{5}$ and $\Lambda_{S}=1.6 \times 10^{4}$, which corresponds to a moderately large value of $\tan \beta=19$. The squark masses for our benchmark point are in the range $750-800 \mathrm{GeV}$, while the mass of the lightest stop is $617 \mathrm{GeV}$. The gluino mass is slightly heavier at $880 \mathrm{GeV}$. The lightest stau mass is $100 \mathrm{GeV}$, just above the bound from direct searches, 


\begin{tabular}{|c|c|c|c|c|c|c|}
\hline Benchmark Point & $\sigma_{p p \rightarrow \tilde{g} \tilde{g}}$ & $\sigma_{p p \rightarrow \tilde{q} \tilde{q}}$ & $\sigma_{p p \rightarrow \tilde{g} \tilde{q}}$ & $\sigma_{p p \rightarrow \tilde{q} \tilde{q}}$ & $\sigma_{p p \rightarrow \tilde{\tau}_{i} \tilde{\tau}_{j}}$ & $\sigma_{p p \rightarrow \chi_{2}^{0} \chi_{1}^{ \pm}}$ \\
\hline PGM2 & 17 & 190 & 164 & 54 & 91 & 49 \\
\hline PGM3 & 16 & 133 & 128 & 34 & 17 & 50 \\
\hline
\end{tabular}

Table 4. This table shows the production rates for the most important processes for the stau (PGM2) and co-NLSP (PGM3) benchmark points at the LHC with $\sqrt{s}=7 \mathrm{TeV}$. All cross-sections are in femtobarns.

and the lightest neutralino mass is $156 \mathrm{GeV}$. The stau-smuon splitting is $29 \mathrm{GeV}$. We now turn to the production cross-sections for this point. As the gluino mass in PGM2 is nearly double that of the neutralino NLSP in points PGM1a and PGM1b, the $p p \rightarrow \tilde{g} \tilde{g}$ cross-section is much smaller. The processes with the largest production cross-sections for the stau NLSP benchmark point PGM2 are shown in table 4 in femtobarns. While the squark production cross-sections are higher than for the PGM1 scenarios, for this point the total number of SUSY events will be about 600, when one includes the processes with smaller contributions. While we have not performed a detailed simulation, the PGM2 point should just be within the range of discovery of the ATLAS detector in the first year of operation [38]. In the stau NLSP scenario one does not expect any missing $E_{T}$ since the pair produced staus will turn up in the calorimeters at the end of the SUSY cascade. From the strong production channels $p p \rightarrow \tilde{g} \tilde{g}$ and $p p \rightarrow \tilde{g} \tilde{q}$ we expect $\geq 2$ jets plus two muon-like objects. In addition we also have significant $\tilde{\tau}$ pair production which should just give two muon-like objects. Together these channels should provide good chances for early SUSY discovery in these scenarios. Finally, single production of neutralinos and charginos in conjuction with a gluino or a squark is negligible.

Next, we discuss the possibility of a stau-neutralino co-NLSP. If we were to decrease $\Lambda_{G}$ very much, this would lead to an unacceptable decrease in the Higgs mass. Therefore we must increase $\Lambda_{S}$ in order to achieve $m_{\tilde{\tau}} \sim m_{\chi_{1}^{0}}$. The co-NLSP point PGM3 has $\Lambda_{G}=1.2 \times 10^{5}, \Lambda_{S}=4.76 \times 10^{4}$ and $\tan \beta=20.5$. The point we have selected has $m_{\tilde{\tau}_{1}}=157 \mathrm{GeV}$ and $m_{\chi_{1}^{0}}=157 \mathrm{GeV}$, with neutralino marginally heavier than the stau. As the scalar mass parameter $\Lambda_{S}$ has increased somewhat, the squark masses are heavier at this point by around $50 \mathrm{GeV}$ compared with the stau NLSP point. The slepton masses are also higher, and the light smuon and selectron masses are $181 \mathrm{GeV}$. The production cross-sections are broadly similar to the stau NLSP case, but somewhat smaller due to the higher masses and more compressed spectrum in this case.

Finally we discuss the stau NLSP point in the many messenger limit, PGM4. This point has the interesting feature that the lightest neutralino is heavier than all the sleptons and sneutrinos. The phenomenology of this scenario has been explored in [39, 40], and includes the presence of many leptons from decay chains leading to the NLSP. It is not possible in PGGM to achieve low enough coloured sparticle masses to have large gluino and squark production cross-sections. The reason for this is as follows. The scalar masses in this gaugino mediated region are generated predominatly by RG running, and take the form

$$
\delta m_{\tilde{f}}^{2} \sim \frac{\alpha}{4 \pi} \Lambda_{G}^{2}
$$


where a summation over the gauge groups is implied. The main constraint on the value of $\Lambda_{G}$ in the gaugino mediated region are the direct search constraints, and specifically the constraint on the mass of the stau. The staus are only weakly interacting, and thus require relatively large values of $\Lambda_{G}$ to evade the direct search constraints. This large $\Lambda_{G}$ is what causes the strongly interacting sparticles to have such large masses. In the full GGM parameter space with three independent gaugino masses one could increase coloured sparticle production by keeping $\Lambda_{G}^{1,2}$ fixed and decreasing $\Lambda_{G}^{3}$. This would leave the slepton, neutralino and chargino masses fixed while decreasing the squark and gluino masses. Accordingly sparticle production at PGM4 at LHC7 is mostly due to direct production of the stau NLSP. This has a cross-section of $62 \mathrm{fb}$. Almost all the produced staus are the NLSP however (the cross section into these being $61.8 \mathrm{fb}$ ). Thus the leptogenic signals due to heavy stau or neutralino decay described in ref. [40] will not be a feature of the LHC at $7 \mathrm{TeV}$ in the pure GGM scenario, and will only appear at higher energies. The main signal in this region for the moment will be an excess of di-muon events, and possibly the displaced vertex signals of NLSP decay inside the detector.

\section{Conclusions}

We have made a survey of the phenomenology of Pure General Gauge Mediation - i.e. in which the $B_{\mu}$ parameter is generated radiatively, with a particular emphasis on its testability in early LHC searches (at $7 \mathrm{TeV}$ ). Five benchmark points were presented: two corresponding to light gluino regions $\left(m_{\tilde{g}} \lesssim 500 \mathrm{GeV}\right.$ with a bino-like neutralino NLSP), two to a stau NLSP and one to stau/neutralino co-NLSP. These benchmark points are representative of the different phenomenology that can occur in the regions of parameter space. We presented a preliminary analysis of the spectrum, production cross sections and branching ratios, which suggests that all of these points can be discovered in the first year of LHC running with appropriate selection cuts. The full set of data in SLHA format for these benchmark points can be found at

$$
\text { http://www.ippp.dur.ac.uk/ SUSY }
$$

We also surveyed and discussed NLSP phenomenology in this set-up, focussing on the possibility of NLSP decays inside the detector in various different schemes of SUSY breaking. Pure GGM with medium to low messenger masses $\left(10^{6-10} \mathrm{GeV}\right)$ can give detectable decays with displaced vertices inside the detector, and hence direct knowledge of the fundamental scale of SUSY breaking.

\section{Acknowledgments}

We thank Yuri Gershtein and Zohar Komargodski for interesting discussions. MJD thanks St John's College, the CET and EPSRC for financial support. SAA and VVK are in receipt of Leverhulme Research Fellowships. 
Open Access. This article is distributed under the terms of the Creative Commons Attribution Noncommercial License which permits any noncommercial use, distribution, and reproduction in any medium, provided the original author(s) and source are credited.

\section{References}

[1] P. Meade, N. Seiberg and D. Shih, General gauge mediation, Prog. Theor. Phys. Suppl. 177 (2009) 143 [arXiv:0801.3278] [SPIRES].

[2] L.M. Carpenter, Surveying the phenomenology of general gauge mediation, arXiv:0812.2051 [SPIRES].

[3] A. Rajaraman, Y. Shirman, J. Smidt and F. Yu, Parameter space of general gauge mediation, Phys. Lett. B 678 (2009) 367 [arXiv: 0903. 0668] [SPIRES].

[4] S. Abel, M.J. Dolan, J. Jaeckel and V.V. Khoze, Phenomenology of pure general gauge mediation, JHEP 12 (2009) 001 [arXiv:0910.2674] [SPIRES].

[5] R. Rattazzi and U. Sarid, Large $\tan \beta$ in gauge-mediated SUSY-breaking models, Nucl. Phys. B 501 (1997) 297 [hep-ph/9612464] [SPIRES].

[6] K.S. Babu, C.F. Kolda and F. Wilczek, Experimental consequences of a minimal messenger model for supersymmetry breaking, Phys. Rev. Lett. 77 (1996) 3070 [hep-ph/9605408] [SPIRES].

[7] S.A. Abel, C. Durnford, J. Jaeckel and V.V. Khoze, Patterns of gauge mediation in metastable SUSY breaking, JHEP 02 (2008) 074 [arXiv:0712.1812] [SPIRES].

[8] G.F. Giudice and R. Rattazzi, Theories with gauge-mediated supersymmetry breaking, Phys. Rept. 322 (1999) 419 [hep-ph/9801271] [SPIRES].

[9] B.C. Allanach, SOFTSUSY: a program for calculating supersymmetric spectra, Comput. Phys. Commun. 143 (2002) 305 [hep-ph/0104145] [SPIRES].

[10] K.I. Izawa, Y. Nomura, K. Tobe and T. Yanagida, Direct-transmission models of dynamical supersymmetry breaking, Phys. Rev. D 56 (1997) 2886 [hep-ph/9705228] [SPIRES].

[11] R. Kitano, H. Ooguri and Y. Ookouchi, Direct mediation of meta-stable supersymmetry breaking, Phys. Rev. D 75 (2007) 045022 [hep-ph/0612139] [SPIRES].

[12] C. Csáki, Y. Shirman and J. Terning, A simple model of low-scale direct gauge mediation, JHEP 05 (2007) 099 [hep-ph/0612241] [SPIRES].

[13] S. Abel, C. Durnford, J. Jaeckel and V.V. Khoze, Dynamical breaking of $U(1)_{R}$ and supersymmetry in a metastable vacuum, Phys. Lett. B 661 (2008) 201 [arXiv:0707.2958] [SPIRES].

[14] S. Abel, J. Jaeckel, V.V. Khoze and L. Matos, On the diversity of gauge mediation: footprints of dynamical SUSY breaking, JHEP 03 (2009) 017 [arXiv:0812.3119] [SPIRES].

[15] Z. Komargodski and D. Shih, Notes on SUSY and R-symmetry breaking in Wess-Zumino models, JHEP 04 (2009) 093 [arXiv: 0902.0030] [SPIRES].

[16] S.A. Abel, J. Jaeckel and V.V. Khoze, Gaugino versus sfermion masses in gauge mediation, Phys. Lett. B 682 (2010) 441 [arXiv:0907.0658] [SPIRES].

[17] C. Cheung, A.L. Fitzpatrick and D. Shih, (Extra)ordinary gauge mediation, JHEP 07 (2008) 054 [arXiv:0710.3585] [SPIRES]. 
[18] E.A. Mirabelli and M.E. Peskin, Transmission of supersymmetry breaking from a 4-dimensional boundary, Phys. Rev. D 58 (1998) 065002 [hep-th/9712214] [SPIRES].

[19] D.E. Kaplan, G.D. Kribs and M. Schmaltz, Supersymmetry breaking through transparent extra dimensions, Phys. Rev. D 62 (2000) 035010 [hep-ph/9911293] [SPIRES].

[20] Z. Chacko, M.A. Luty, A.E. Nelson and E. Ponton, Gaugino mediated supersymmetry breaking, JHEP 01 (2000) 003 [hep-ph/9911323] [SPIRES].

[21] C. Csáki, J. Erlich, C. Grojean and G.D. Kribs, 4D constructions of supersymmetric extra dimensions and gaugino mediation, Phys. Rev. D 65 (2002) 015003 [hep-ph/0106044] [SPIRES].

[22] M. McGarrie and R. Russo, General gauge mediation in 5D, Phys. Rev. D 82 (2010) 035001 [arXiv: 1004.3305] [SPIRES].

[23] M. McGarrie, General gauge mediation and deconstruction, arXiv:1009.0012 [SPIRES].

[24] D. Green, A. Katz and Z. Komargodski, Direct gaugino mediation, arXiv:1008.2215 [SPIRES].

[25] B. Altunkaynak, M. Holmes, P. Nath, B.D. Nelson and G. Peim, SUSY discovery potential and benchmarks for early runs at $\sqrt{s}=7 \mathrm{TeV}$ at the LHC, arXiv:1008.3423 [SPIRES].

[26] H. Baer, V. Barger, A. Lessa and X. Tata, Capability of LHC to discover supersymmetry with $\sqrt{s}=7 \mathrm{TeV}$ and $1 \mathrm{fb}^{-1}$, JHEP 06 (2010) 102 [arXiv:1004.3594] [SPIRES].

[27] D.S.M. Alves, E. Izaguirre and J.G. Wacker, It's on: early interpretations of ATLAS results in jets and missing energy searches, arXiv: 1008.0407 [SPIRES].

[28] W. Beenakker, R. Hopker and M. Spira, PROSPINO: a program for the PROduction of Supersymmetric Particles In Next-to-leading Order QCD, hep-ph/9611232 [SPIRES].

[29] T. Plehn, Prospino2.0 http://www.thphys.uni-heidelberg.de/ plehn.

[30] P. Meade, M. Reece and D. Shih, Prompt decays of general neutralino NLSPs at the Tevatron, JHEP 05 (2010) 105 [arXiv: 0911.4130] [SPIRES].

[31] CDF collaboration, T. Aaltonen et al., Search for supersymmetry with gauge-mediated breaking in diphoton events with missing transverse energy at CDF II,

Phys. Rev. Lett. 104 (2010) 011801 [arXiv:0910.3606] [SPIRES].

[32] D0 collaboration, V.M. Abazov et al., Search for diphoton events with large missing transverse energy in $6.3 \mathrm{fb}^{-1}$ of pp collisions at $\sqrt{s}=1.96 \mathrm{TeV}$, arXiv:1008.2133 [SPIRES].

[33] D0 collaboration, V.M. Abazov et al., Search for associated production of charginos and neutralinos in the trilepton final state using $2.3 \mathrm{fb}^{-1}$ of data, Phys. Lett. B 680 (2009) 34 [arXiv: 0901.0646] [SPIRES].

[34] CDF collaboration, R. Forrest, Search for supersymmetry in $p \bar{p}$ collisions at $\sqrt{s}=1.96 \mathrm{TeV}$ using the trilepton signature of chargino-neutralino production, arXiv:0910.1931 [SPIRES].

[35] S. Ambrosanio, G.D. Kribs and S.P. Martin, Three-body decays of selectrons and smuons in low-energy supersymmetry breaking models, Nucl. Phys. B 516 (1998) 55 [hep-ph/9710217] [SPIRES].

[36] H. Murayama and Y. Nomura, Gauge mediation simplified, Phys. Rev. Lett. 98 (2007) 151803 [hep-ph/0612186] [SPIRES].

[37] K.A. Intriligator, N. Seiberg and D. Shih, Dynamical SUSY breaking in meta-stable vacua, JHEP 04 (2006) 021 [hep-th/0602239] [SPIRES]. 
[38] A.R. Raklev, Massive metastable charged (S)particles at the LHC, Mod. Phys. Lett. A 24 (2009) 1955 [arXiv:0908.0315] [SPIRES].

[39] A. De Simone, J. Fan, M. Schmaltz and W. Skiba, Low-scale gaugino mediation, lots of leptons at the LHC, Phys. Rev. D 78 (2008) 095010 [arXiv: 0808.2052] [SPIRES].

[40] A. De Simone, J. Fan, V. Sanz and W. Skiba, Leptogenic supersymmetry, Phys. Rev. D 80 (2009) 035010 [arXiv: 0903.5305] [SPIRES]. 